Christine M. Freitag

Christina Kleser

Alexander von Gontardf

\section{Imitation and language abilities in adolescents with Autism Spectrum Disorder without language delay}

Accepted: 6 February 2006

Published online: 22 March 2006
C.M. Freitag, MD ( $\square)$

Dept. of Child and Adolescent Psychiatry Saarland University Hospital 66421 Homburg (Saar), Germany

Tel.: +49-6841/16-24388

Fax: +49-6841/16-24397

E-Mail: christine.freitag@uniklinikumsaarland.de

\section{Abstract}

Introduction: Difficulties imitating gestures have been found in several studies in children and adolescents with Autism Spectrum Disorders (ASD). It has been hypothesized that imitation abilities might be precursors of language abilities in young children with autism. No study on imitation and language abilities in adolescents with ASD has been performed to date.

Methods: Fifteen male adolescents with ASD, 16 male and 13 female controls were compared regarding imitation abilities of upper and lower facial movements, and language skills as assessed by the pragmatic rating scale and the Aachen Aphasia test (AAT).

Results: Autism Spectrum Disorder subjects showed reduced imitation abilities of facial movements and non-meaningful combined hand-and-finger ges- tures. Regarding language, ASD subjects showed difficulties in AAT spontaneous speech measures and reduced pragmatic language abilities. Correlations of imitation and language measures differed between ASD, male and female controls.

Conclusion: The weak and differential correlations of imitation and language measures in the three comparison groups might imply a differential organization of language and imitation networks in the three comparison groups. Pragmatic abilities, which are a central feature in ASD, were not related to imitation abilities. Therefore, imitation and language abilities in ASD might not be as closely correlated as previously expected.

Key words autism spectrum disorder - imitation - language - apraxia

\section{Introduction}

Autism spectrum disorders (ASD) are pervasive developmental disorders, characterized by three core deficits, i.e., social interaction and communication problems, and restrictive, stereotyped and repetitive behaviours and interests $[1,54]$. There has been considerable effort to elucidate a core neuropsychological finding, which might be equally related to the three areas of impairment in ASD and might therefore lead to a better understanding of the disorder. Three reviews $[48,50,55]$ have emphasized the pervasive nature of imitation deficits in children and adolescents with ASD. Regarding the reviewed studies, four types of imitative actions were compared: action upon an object, meaningful and non-meaningful gestures, and action upon a substituted object. A meta-analysis of 
the reported studies [55] has shown that imitation of non-meaningful gestures was most impaired, followed by meaningful gestures and actions upon an object.

It has been discussed that deficits in the ability to imitate other people's gestures and actions might be caused either by an executive function deficit (for review: 44) or by lower motor abilities in children and adolescents with ASD [21]. However, Rogers et al. [46] found only partial evidence for an underlying executive function deficit in a group of 11-21-year-old high-functioning persons with ASD and controls, suffering from dyslexia or other child psychiatric disorders. The same study did rule out an underlying symbolic deficit, as postulated by the meta-representational theory (for review: 22). Regarding motor abilities, several studies have shown that motor abilities only in small parts accounted for imitative deficits in autism [21, 46, 51]. The discussed perception deficit in ASD has not yet been assessed with regard to imitation abilities [45].

Among others, the notion of an imitation deficit in children and adolescents with ASD is of interest, as it might provide an explanation of the delayed language acquisition in children with autism. It has been hypothesized that imitation abilities of newborns are a prerequisite for the development of language and communication in toddlers [38]. An association of abilities in imitating body movements and expressive language level has been found in a longitudinal study of 2-3 year-old children with autism at a 1- and 2year follow-up $[52,53]$. In a cross-sectional study on 2-4-year-old children with autism, however, no correlation of imitation and language abilities in children with autism was present, whereas in the comparison group of children with developmental delay, a strong correlation of manual imitation, object imitation and oral imitation with expressive language skills was found [47]. This might point to a qualitatively different development of imitation and language abilities in children with ASD.

Regarding children and adolescents with ASD without language delay, like Asperger Syndrome (AS) and High-functioning autism (HFA) without language delay, language acquisition takes place within normal developmental limits. However, there is some evidence that, despite normal language acquisition and high verbal IQ measures in the Wechsler Intelligence Scales, receptive and expressive language skills might be impaired in adolescents and adults with ASD without language delay $[17,24]$. Language and imitation abilities have not yet been assessed together in adolescents with ASD without language delay.

From the assessment of adult patients with brain lesions, it is well known that imitation abilities of meaningless gestures are reduced in patients with ideomotor apraxia $[19,20,31]$. Apraxia and aphasia often occur together in the same patient, however, in some patients, a dissociation of symptoms can be found. This suggests that praxis and language are mediated by overlapping but differing networks [43]. The discovery of "mirror neurons" in primates, which are active during observation and during performance of the same motor task [26] has provided further insight in the biological basis for imitation deficits. Functional magnetic resonance tomography (fMRI) studies in humans suggest that Broca's area in the left inferior frontal cortex as well as the right parietal cortex [26] and the superior temporal sulcus bilaterally [27, 34] are activated during observation and imitation of finger movements. Another study found evidence for a common network for both face and hand imitation, consisting of Broca's area, bilateral dorsal and ventral premotor areas, right superior temporal gyrus, supplementary motor area, posterior temporo-occipital cortex and cerebellar areas [35]. Some of these areas are in close anatomical proximity to cortical language processing areas as Broca's and Wernicke's areas.

Regarding the possible functional overlap between imitation and language abilities and their impairment in ASD, a study of imitative abilities of facial and nonmeaningful hand and finger movements and language abilities in male adolescents with ASD without language delay and male and female controls was performed. These three groups were chosen to obtain sex differences in healthy controls and to compare these with the differences between male ASD and male controls, as ASD by some authors are considered to be on the extreme of the male behaviour [5].

It was hypothesized that imitation abilities of upper and lower facial movements, and of non-meaningful hand and finger postures as well as language abilities were impaired in the ASD subjects compared to male and female controls but not between controls. Regarding the full sample, a correlation of imitation and language abilities was postulated. As a common network for both face and hand imitation has been postulated, it was hypothesized that all imitation measures showed a positive correlation in the full sample and in cases and controls separately. Further, it was hypothesized that imitation abilities of lower facial movements showed the strongest association with expressive language abilities as articulation and prosody, repetition of syllables, words and sentences, and naming of articles, situations and actions as measured by the Aachen Aphasia test (AAT; [25]) due to the involvement of the same neuromuscular structures. Due to the postulated involvement of mirror neurons in finger and hand imitation and the close anatomical proximity to Broca's area, however, it was further hypothesized that imitation of finger and hand movements were correlated with similar 
expressive language abilities and additionally the AAT spontaneous speech measure phonologic structure.

\section{Methods}

\section{Sample}

Three groups of adolescents were included in the study: 15 adolescents with ASD, 16 male and $13 \mathrm{fe}-$ male controls, aged 14-22 years. Informed consent was obtained by the adolescents and their parents. The study design was approved by the local ethics committee.

Inclusion criteria for the ASD group were as follows: Autism criteria were met in the Autism Diagnostic Interview-revized (ADI-R; 37) with the parents, and in direct observation by the Autism Observation Schedule generic (ADOS-G; 36). Additionally, a thorough review of medical records was performed. Language development was within normal limits (first words before age 24 months, short sentences before age 33 months). Eleven subjects with ASD were diagnosed as HFA due to other developmental problems before age 3 years according to ADI-R criteria (item 94), four subjects were diagnosed with AS. Full scale IQ was $>70$ on the German version of the Wechsler Intelligence Scale for children (WISC), version III or on the Wechsler Adult Intelligence Scale (WAIS) - revised.

Exclusion criteria for all groups were: history of cerebral palsy; congenital anomaly of the central nervous system; history of schizophrenia; known genetic syndrome; history of focal epilepsy; tuberous sclerosis, neurofibromatosis. Initially, 18 adolescents with HFA or AS were assessed. Three HFA patients were excluded from the study due to an ArnoldChiari-Malformation, a balanced translocation in cytogenetic assessment and premature birth with a birth weight of $1500 \mathrm{~g}$.

For the ASD adolescents, data on pregnancy and development were obtained by their parents. In controls, birth problems, prematurity and history of psychiatric disorders were excluded by asking the adolescents themselves. All adolescents completed the Youth Self-report (YSR) or Young Adult Self-report (YASR); parents completed the Child Behaviour Checklist (CBCL). American norms were used for YSR and YASR [2, 3]; German norms were used for the CBCL [4]. Intelligence was measured by the German version of the WISC, version III, with norms from 2000 or of the WAIS revised, with norms from 1991. Handedness was determined by the Edinburgh Handedness inventory [41]. All subjects had normal or corrected to normal vision.

\section{Imitation assessment}

Imitation of facial movements was assessed by lower and upper facial apraxia tasks as published by Bizzozero et al. [8]. Imitation of upper facial movements was assessed by nine items representing an action (closing eyes, wrinkling nose, etc.). Imitation of lower facial movements was assessed by 29 items (sticking out tongue, open mouth, etc.). Each task was first demonstrated by the examiner, and probands were asked to imitate it. Two demonstrations and two verbal instructions were maximally allowed for. Probands scored 4 points if they managed to correctly imitate the task after the first presentation, 3 points after the second presentation, 2 points after the first verbal instruction and 1 point after the second verbal instruction. If probands performed arbitrary or incomplete movements, they were scored 0 points. Summary scores were calculated separately for upper (maximum score 36) and lower facial movements (maximum score 116). For each gesture, correct mirror imitation was scored separately.

Imitation of non-meaningful hand, finger and hand-finger gestures was assessed by ideomotor apraxia tasks as published by Goldenberg [19]. Imitation of hand gestures required probands to copy different positions of the hand relative to the head, while the configuration of the fingers remained invariant. For imitation of finger gestures, probands were asked to replicate different configurations of the fingers. The position of the whole hand relative to the body was not considered for scoring. Combined gestures required both a defined position of the hand relative to the body and a defined configuration of the fingers. The examiner stood opposite of the proband and demonstrated the gestures in a mirror fashion. For a correct imitation after the first trial, 2 points were scored. 1 point was scored for correct imitation after the second trial. There were 14 items for each type of gesture resulting in a maximum score of 28 for each type of gesture. Half of the gestures were performed with the right hand and half of the gestures with the left hand. For each gesture, correct mirror imitation was scored separately.

\section{Assessment of motor performance}

To control for lower motor performance speed in adolescents with AS, repetitive and alternating hand and repetitive and sequential finger movement speed was assessed by the Zurich Neuromotor Assessment battery (ZNA, 33). Only alternating hand movements differed between groups and were therefore controlled for. 


\section{Language assessment}

In addition to the verbal IQ measures of the WISC and WAIS, the Aachen Aphasia test (AAT, [25]) and the Pragmatic Rating Scale - working draft (PRS; [32]) were performed.

The AAT is a standardized assessment with German norms for probands aged 14 years and older to assess detailed symptoms of aphasia. It consists of six tests. (1) Evaluation of spontaneous speech with six subscores, ranging from 0 to $5,(2)$ token test, ranging from 0 to 100 , (3) repetition of syllables, words and sentences, ranging from 0 to 100 , (4) written language, including reading and writing, ranging from 0 to 100 , (5) naming of articles, situations and actions, ranging from 0 to 100 , (6) listening and reading comprehension, ranging from 0 to 100 .

The PRS consists of 24 items covering pragmatic abilities. Items are rated between 0 (appropriate pragmatics) and 2 (atypical pragmatic behaviour). Maximum summary score is 48 . The items were rated by the examiner after the assessment of imitation, AAT, WISC/WAIS, and/or ADOS-G (in ASD probands only). Forty of the PRS assessments were rated from video by a second rater. Interrater reliability of the summary score as assessed by intraclass correlation was 0.92 .

\section{Statistical analysis}

Descriptive data were compared by analysis of variance (ANOVA) and Fisher's exact test. Imitation and language abilities in the three comparison groups were compared by analysis of covariance (ANCOVA) models, with verbal IQ, performance IQ and age as covariates, and case, male control or female control status as the predictive variable of interest. In the
ANCOVA for hand and hand-finger imitation tasks, additionally, alternating hand movement measures from the ZN were included as covariates to control for motor differences between groups. Data were multivariate normally, in case of the first six AAT subscores, approximately multivariate normally distributed. Findings were considered to be significant if the lowest $p$-value was $p<0.0029$, the second lowest $p<0.0031$, etc. according to a BonferroniHolm correction for multiple testing. Planned contrasts were calculated for ASD vs. male controls and male vs. female controls.

Correlations of language and imitation measures were assessed by multiple regression models in the full sample, with language measures as dependent and imitation measures as independent variables of interest. Verbal and performance IQ, age and casecontrol status were included as covariates. Two models were compared for each measure, one without and one with an additional interaction term for imitation measures and case-control status to evaluate differential correlation of language and imitation measures between groups. When an association was found in the full sample, this association was assessed in the three comparison groups separately. Correlations between face and hand/finger imitation measures were assessed by similar multiple regression procedures. Due to low sample size, the evaluation of correlations of language and imitation measures was not adjusted for multiple testing.

\section{Results}

Descriptive data on the adolescents with ASD and male and female controls are shown in Table 1. ASD

Table 1 Descriptive data of the three comparison groups

\begin{tabular}{|c|c|c|c|c|}
\hline & $\begin{array}{l}\text { Autism Spectrum } \\
\text { Disorder, } N=15, \\
\text { mean (SD) }\end{array}$ & $\begin{array}{l}\text { Male controls, } \\
N=16 \\
\text { mean (SD) }\end{array}$ & $\begin{array}{l}\text { Female controls, } \\
N=13, \\
\text { mean (SD) }\end{array}$ & $\begin{array}{l}\text { ANOVA statistic } \\
F \text { value (DF) } \\
p \text {-value }\end{array}$ \\
\hline Verbal IQ & $108.3(19.0)$ & $111.3(21.3)$ & $116.5(8.3)$ & $0.8(2,41) p=0.464$ \\
\hline Performance IQ & $91.0(20.7)$ & $100.0(20.5)$ & $117.4(11.0)$ & $7.4(2,41) p=0.002$ \\
\hline Full scale IQ & $100.1(17.8)$ & $107.3(21.6)$ & $118.5(7.1)$ & $4.1(2,41) p=0.024$ \\
\hline Age in years & $16.5(2.4)$ & $17.9(1.6)$ & $18.4(0.9)$ & $4.4(2,41) p=0.019$ \\
\hline CBCL externalising score & $56.2(7.1)$ & $52.1(7.8)$ & $47.7(7.9)^{*}$ & $4.2(2,40) \boldsymbol{p}=\mathbf{0 . 0 2 3}$ \\
\hline $\mathrm{CBCL}$ internalising score & 70.7 (7.9) & $51.2(6.1)$ & $50.9(8.7)^{*}$ & $33.5(2,40) p<0.0001$ \\
\hline CBCL Total problem score & $68.5(7.9)$ & $52.5(7.6)$ & $49.1(7.2)^{*}$ & $26.3(2,40) p<0.0001$ \\
\hline Y(A)SR externalising score & $52.4(6.9)^{*}$ & $52.6(9.2)$ & $47.9(10.9)$ & $1.2(2,40) p=0.326$ \\
\hline Y(A)SR internalising score & $63.4(8.2)^{*}$ & $53.6(12.5)$ & $52.3(13.3)$ & $3.9(2,40) p=0.030$ \\
\hline Y(A)SR Total problem score & $\begin{array}{c}61.7(7.5)^{*} \\
n(\%)\end{array}$ & $\begin{array}{c}54.4(9.7) \\
n(\%)\end{array}$ & $\begin{array}{c}50.2(14.3) \\
n(\%)\end{array}$ & $4.0(2,40) \boldsymbol{p}=\mathbf{0 . 0 2 5}$ \\
\hline Right handed & $13(86.7 \%)$ & $15(93.8 \%)$ & $10(76.9 \%)$ & Fisher's exact test $p=0.406$ \\
\hline
\end{tabular}

$\mathrm{DF}$, degrees of freedom; SD, standard deviation

*Information missing on one individual 
probands were group matched with regard to verbal IQ. In the female controls, full scale and performance IQ were highest, and ASD probands were younger than controls. Therefore, these data were controlled for in further analyses. Regarding subtests of the WISC or WAIS controlled for age and full scale IQ differences, girls showed least information and arithmetic skills but best performance in the symbol search test compared to the two other groups $(p<0.05)$. ASD probands performed best in the subtest digit span compared to male controls $(p<$ $0.05)$. Socio-economic status did not differ between groups.

\section{Imitation abilities}

In Table 2, imitation abilities of upper and lower face movements and of non-meaningful hand and finger gestures in the three comparison groups are shown. Imitation abilities of upper and lower facial movements differed between groups. In post-hoc analyses, this was due to a difference of ASD and male (upper facial movements: $F=7.9,1 \mathrm{DF}, p=0.008$; lower facial movements: $F=15.1,1 \mathrm{DF}, p \leq 0.0001)$ and female (upper facial movements: $F=11.1,1 \mathrm{DF}$, $p=0.002$; lower facial movements: $F=21.4,1 \mathrm{DF}$, $p<0.0001)$ controls. Male and female controls did not differ (upper facial movements: $F=1.1,1 \mathrm{DF}$, $p<0.297$; lower facial movements: $F=0.2,1 \mathrm{DF}$, $p=0.664)$. There was no difference regarding the amount of movements imitated in a mirror fashion compared to non-mirror imitation between groups. No correlation of verbal or performance IQ measures with imitation abilities of face movements was found.

Imitation of non-meaningful hand or finger gestures did not differ between groups. However, the more complex hand- and finger-gestures were more difficult for ASD (Table 2). Post-hoc analysis resulted in lower hand-finger imitation abilities in ASD compared to male $(F=15.8,1 \mathrm{DF}, p=0.0003)$ and female $(F=16.5,1 \mathrm{DF}, p=0.0002)$ controls. Male and female controls did not differ with regard to hand-finger imitation gestures $(F=0.2,1 \mathrm{DF}, p=0.698)$. Performance IQ was correlated with the imitation of hand gestures (estimate 0.06, SE 0.02, $t$-value 2.9, $p=0.006$ ) in the full sample. No other correlation of verbal or performance IQ measures or speed of alternating hand movements with hand or finger imitation abilities was found. The score of correctly imitated left vs. right hand and finger movements did not differ in each of the comparison groups. Between groups, there was no difference regarding the amount of movements imitated in a mirror fashion compared to a non-mirror fashion.

In the full sample, an association of non-meaningful hand (estimate 1.17, SE 0.57, $t$-value 2.1, $p=0.047$ ), finger (trend: estimate $0.71, \mathrm{SE} 0.36, t$-value $2.0, p=0.059$ ) and hand-and-finger movements (estimate 0.96, SE $0.35, t$-value $2.8, p=0.009$ ) with imitation of lower facial movements was found. No association of hand or finger imitation and upper face movement imitation abilities was found.

\section{Language abilities}

In Table 3, language abilities in the three comparison groups are shown. Differences were found with regard to the four AAT spontaneous speech measures: communicative behaviour, articulation and prosody, semantic structure and syntactic structure. Post-hoc analyses did show differences between ASD and male controls (communicative behaviour: $F=8.4,1 \mathrm{DF}$, $p=0.006$; articulation and prosody: $F=87.5,1 \mathrm{DF}$, $p<0.0001$; semantic structure: $F=12.0,1 \mathrm{DF}, p=0.001$; syntactic structure: $F=11.9,1 \mathrm{DF}, p=0.001$ ) and female controls (communicative behaviour: $F=13.7,1 \mathrm{DF}$, $p=0.0007$; articulation and prosody: $F=69.3,1 \mathrm{DF}$,

Table 2 Imitation of facial movements and non-meaningful hand and finger movements in Autism Spectrum Disorder and male and female controls

\begin{tabular}{|c|c|c|c|c|}
\hline Imitation tasks & $\begin{array}{l}\text { Autism Spectrum Disorder, } \\
N=15 \text {, } \\
\text { adjusted mean* }(95 \% \text { Cl) }\end{array}$ & $\begin{array}{l}\text { Male controls, } \\
N=16 \\
\text { adjusted mean }{ }^{*}(95 \% \text { Cl) }\end{array}$ & $\begin{array}{l}\text { Female controls, } \\
N=13, \\
\text { adjusted mean* }(95 \% \mathrm{Cl})\end{array}$ & $\begin{array}{l}\text { Statistic } \\
F \text { value (DF) } \\
p \text {-value }\end{array}$ \\
\hline Upper facial movements & $27.6(25.4-29.8)$ & $31.8(29.8-33.8)$ & $33.4(31.0-35.8)$ & $6.2(2)^{* *} \boldsymbol{p}=\mathbf{0 . 0 0 5}$ \\
\hline Lower facial movements & $96.1(92.0-100.2)$ & $109.7(106.1-113.4)$ & $111.0(106.5-115.4)$ & $15.1(2)^{* *} p<0.0001$ \\
\hline Hand posture & $25.6(24.5-26.8)$ & $27.1(26.0-28.1)$ & $26.4(25.2-27.6)$ & $1.7(2)^{* * *} p=0.201$ \\
\hline Finger posture & $22.4(20.6-24.1)$ & $24.7(23.1-26.3)$ & $24.7(22.8-26.6)$ & $2.2(2)^{* *} p=0.120$ \\
\hline Hand-and-finger posture & $19.5(17.7-21.4)$ & $24.7(23.0-26.4)$ & $25.2(23.3-27.2)$ & $10.5(2)^{* * *} p=0.0003$ \\
\hline
\end{tabular}

DF: degrees of freedom; Cl: confidence interval

*Adjusted for age, performance and verbal IQ. For assessment of hand and hand-and-finger imitation tasks, additional adjustment for speed of alternating hand movements

**Model $\mathrm{DF}=5$; error $\mathrm{DF}=38$

****Model DF $=7$; error DF $=36$ 
Table 3 Language abilities: Aachen Aphasia Test and Pragmatic Rating Scale in Autism Spectrum Disorder and male and female controls

\begin{tabular}{|c|c|c|c|c|}
\hline Language measure & $\begin{array}{l}\text { Autism Spectrum Disorder, } \\
N=15 \text {, adjusted } \\
\text { mean* }(95 \% \mathrm{Cl})\end{array}$ & $\begin{array}{l}\text { Male controls, } \\
N=16, \text { adjusted } \\
\text { mean* }(95 \% \text { Cl })\end{array}$ & $\begin{array}{l}\text { Female controls, } \\
N=13 \text { adjusted } \\
\text { mean }^{*}(95 \% \mathrm{Cl})\end{array}$ & $\begin{array}{l}\text { Statistic } F \text { value } \\
\text { (DF), } p \text {-value }\end{array}$ \\
\hline AAT - spontaneous speech: communicative behaviour & $4.2(3.9-4.4)$ & $4.7(4.5-4.9)$ & $4.9(4.7-5.2)$ & $7.4(2){ }^{* *} p=0.002$ \\
\hline AAT - spontaneous speech: articulation and prosody & $3.5(3.3-3.7)$ & $4.9(4.7-5.2)$ & $5.0(4.7-5.3)$ & $51.3(2) * * p<0.0001$ \\
\hline AAT - spontaneous speech: automated speech & $4.8(4.6-5.0)$ & $5.0(4.8-5.2)$ & $5.0(4.8-5.2)$ & $0.9(2)^{* *} p=0.429$ \\
\hline AAT - spontaneous speech: semantic structure & $4.5(4.3-4.7)$ & $5.0(4.8-5.2)$ & $5.0(4.8-5.2)$ & $6.8(2)^{* *} p=0.003$ \\
\hline AAT - spontaneous speech: phonologic structure & $5.0(4.9-5.1)$ & $4.9(4.8-5.0)$ & $4.9(4.8-5.1)$ & $0.25(2)^{* *} p=0.780$ \\
\hline AAT - spontaneous speech: syntactic structure & $4.4(4.2-4.7)$ & $5.0(4.8-5.2)$ & $4.9(4.7-5.2)$ & $6.3(2)^{* *} p=0.004$ \\
\hline AAT - token test & $70.7(69.3-72.2)$ & $69.5(68.2-70.8)$ & $71.4(69.8-72.9)$ & $2.0(2)^{* *} p=0.153$ \\
\hline AAT - repetition of syllables, words and sentences & $68.8(67.2-70.5)^{* * *}$ & $71.1(69.6-72.6)^{* * *}$ & $71.0(69.2-72.8)$ & $2.3(2)^{* *} p=0.113$ \\
\hline AAT - written language & $72.9(70.3-75.5)$ & $72.1(69.8-74.5)$ & $75.3(72.5-78.1)$ & $1.5(2)^{* *} p=0.236$ \\
\hline AAT - naming of articles, situations and actions & $65.2(63.0-67.3)$ & $68.4(66.5-70.4)$ & $70.2(67.9-72.6)$ & $4.7(2)^{* *} p=0.015$ \\
\hline AAT - comprehension & $71.4(68.9-73.9)$ & $68.8(66.6-71.1)$ & $70.1(67.4-72.9)$ & $1.2(2)^{* *} p=0.306$ \\
\hline Pragmatic Rating Scale Summary Score & $17.7(15.9-19.5)$ & $3.8(2.2-5.4)$ & $2.8(0.8-4.8)$ & $78.2(2)^{* *} p<0.0001$ \\
\hline
\end{tabular}

AAT, Aachen Aphasia Test; DF, degrees of freedom; Cl: confidence interval

${ }^{*}$ Adjusted for age, performance and verbal IQ

** Model DF $=5$; error DF $=38$

***Planned contrast ASD vs. male controls: $F=4.1,1 \mathrm{DF}, p=0.049$

$p<0.0001$; semantic structure: $F=8.7,1 \mathrm{DF}, p=0.006$; syntactic structure: $F=6.4,1 \mathrm{DF}, p=0.016)$, but not between male and female controls (communicative behaviour: $F=1.9,1 \mathrm{DF}, p=0.175$; articulation and prosody: $0.2,1 \mathrm{DF}, p=0.669$; semantic structure: $F=0.0$, $1 \mathrm{DF}, \quad p=0.988$; syntactic structure: $F=0.2,1 \mathrm{DF}$, $p=0.847)$. With regard to the other AAT subtests, groups did not differ after correction for multiple testing. However, a nominal significant difference was found for naming of articles, situations and actions, which differed between ASD and male $(F=5.1,1 \mathrm{DF}$, $p=0.030)$ and female $(F=8.9,1 \mathrm{DF}, p=0.005)$ controls; and with regard to repetition of syllables, words and sentences, which differed between ASD and male controls $(F=4.1,1 \mathrm{DF}, p=0.049)$. Regarding pragmatic language abilities, again ASD did show much lower pragmatic abilities than male $(F=131.2,1 \mathrm{DF}$, $p<0.0001)$ and female controls $(F=108.4,1 \mathrm{DF}$, $p<0.0001)$ controls. Male and female controls did not differ regarding pragmatic language abilities $(F=0.6$, $1 \mathrm{DF}, p=0.443$ ).

In the full sample, verbal IQ was strongly related to the AAT measures syntactic structure (estimate 0.01$, SE $0.00, t$-value $2.9, p=0.006)$, token test (estimate 0.14 , SE $0.03, t$-value 5.1, $p<0.0001$ ), repetition of syllables, words and sentences (estimate 0.20 , SE $0.03, t$-value $6.5, p<0.0001$ ), written language (estimate $0.24, \mathrm{SE} 0.05, t$-value $4.9, p<0.0001$ ), naming (estimate $0.20, \quad S E 0.04, t$-value 5.0, $p<0.0001$ ) and comprehension (estimate 0.30, SE $0.05, t$-value $6.4, p<0.0001)$. Verbal IQ abilities, however, did not correlate with pragmatic abilities (estimate $-0.05, \mathrm{SE} 0.03, t$-value $-1.4, p=0.183$ ). No correlation between performance IQ and verbal abilities was found.

\section{Prediction of language abilities by imitation abilities of upper and lower facial movements}

Imitation abilities of upper facial movements predicted the AAT spontaneous speech measure articulation and prosody (estimate 0.04, SE 0.02, $t$-value 2.2, $p=0.032$ ) in the full sample, independent of casecontrol status. In a separate analysis in each of the three comparison groups, an association of imitation abilities of upper facial movements and the AAT spontanueous speech measure articulation and prosody was found in the male control group (estimate 0.04 , SE $0.02, t$-value 2.3, $p=0.045)$, but not in the ASD group (estimate 0.04 , SE $0.04, t$-value $0.9, p=0.382$ ). Due to low variability in the female control group, a separate analysis in this group could not be performed.

Imitation abilities of lower facial movements predicted the AAT spontaneous speech measure automated speech (estimate 0.02, SE $0.01, t$-value 2.1, $p=0.043$ ), and the AAT subscales naming of articles, situations and actions (estimate $0.21, \mathrm{SE} 0.08, t$-value 2.7, $p=0.011$ ) and comprehension (estimate 0.20 , SE $0.09, t$-value $2.1, p=0.039$ ) in the full group. Due to low variability in male and female controls, a separate analysis in each of the three comparison groups could not be performed for the AAT spontaneous speech measure automated speech. Regarding the AAT subscale naming of articles, situations and actions, a trend towards association of lower facial movements with this subscale was found in the ASD group (estimate $0.17, \mathrm{SE} 0.08, t$-value $2.1, p=0.062$ ), but not in the male and female controls. Regarding the AAT subscale comprehension, a trend towards association of lower facial movements was found in the male 
control group (estimate $0.52, \mathrm{SE} 0.27, t$-value 1.9 , $p=0.082$ ), but not in the ASD or the female control group.

No association of pragmatic abilities with imitation abilities of facial movement measures was found.

\section{Prediction of language abilities by imitation abilities of non-meaningful hand and finger gestures}

An interaction of the imitation of non-meaningful hand gestures with case-control status was found regarding the prediction of the AAT measure repetition of syllables, words and sentences $(F=4.5,1 \mathrm{DF}$, $p=0.017)$. This was due to differential association in male and female controls in separate analyses. In males, a positive (estimate $0.78, \mathrm{SE} 0.34 ; t$-value 2.3 , $p=0.043)$ and in females, a negative association (estimate -2.07 , SE $0.71 ; t$-value $-2.9, p=0.019$ ) was found. In the ASD group, no association of imitation of non-meaningful hand gestures with repetition of syllables, words and sentences was detected (estimate 0.21 , SE 0.45 ; $t$-value $0.5, p=0.644$ ).

The imitation of non-meaningful finger gestures negatively predicted repetition of syllables, words and sentences in the full sample (estimate -0.37 , SE 0.17 ; $t$-value $-2.3, p=0.028)$. Additionally, an interaction term of finger imitation and case-control status was found $(F=3.6,2 \mathrm{DF}, p=0.037)$. This was due to a negative association in female controls only (estimate -1.05 , SE $0.34 ; t$-value $-3.1, p=0.014)$. No association was found in the ASD (estimate -0.27 , SE $0.29, t$-value $-0.9, p=0.871$ ) or in the male control group (estimate 0.22 , SE $0.23, t$-value $1.0, p=0.360$ ).

Imitation abilities of non-meaningful hand-andfinger gestures did not predict any of the language measures. No association of pragmatic abilities with imitation abilities of hand/finger gestures was found.

\section{Discussion}

As expected, imitation and language abilities differed between ASD and male and female controls, but not between male and female controls. Language and imitation abilities, however, were differentially correlated in the three comparison groups.

The ASD subjects of our study were most impaired with regard to the imitation of upper and lower facial movements and of non-meaningful hand-and-finger postures. These findings differ slightly from the findings of Rogers et al. [46] who found differences with regard to the imitation of non-meaningful single hand gestures but no differences with regard to nonmeaningful single face gestures in a group of 15-year- old adolescents with ASD, possibly due to low variability of scores in their study. Similar to our study Green et al. [21] reported impaired performance in a gesture test of representational and non-representational gestures in 6-10-year-old children with AS compared to children with specific developmental disorder of motor function. In a group of children with autism with a mean mental age of $7 ; 8$ years, Smith and Bryson [51] reported difficulties in imitating unimanual postures of the deaf alphabet despite no problems in gesture recognition and gesture memory between groups. This shows, that in older adolescents and young adults with ASD, difficulties in the imitation of facial movements and of non-meaningful hand-and-finger postures are still present, and maturation processes of involved brain structures seem not to be able to fully compensate these imitation problems.

As the ASD subjects in our study did not have more difficulties than controls to recognize and imitate hand or finger gestures, problems with imitation of combined hand-and-finger gestures seem not to be related to a general visuo-spatial recognition deficit but more so to the complexity of the task $[6,39]$. It might be possible that difficulties in the imitation of the hand-and-finger imitation task might be due to an underlying perceptual deficit with difficulties using the contextual information of the face in the handand-finger imitation task $[13,45]$. However, the assessment of an underlying perceptual deficit with regard to the imitation of hand and finger postures has to be assessed in a more specific study designed to control for local vs. global processing.

As hand and finger imitation stimuli did not consist of movements, but of a posture that had to be imitated, the reported biological motion processing difficulties of persons with autism [9] are unlikely to explain the hand-finger imitation deficit. Similarly, as at least four (44\%) of the upper facial and sixteen $(55 \%)$ of the lower facial movements were imitated correctly by every single ASD subject, problems with imitation of facial movements cannot be fully explained by a recognition deficit of faces or facial movements in ASD [12], as this should have resulted in even worse performance of facial movement imitation. However, impairments in recognition of faces and facial movements cannot be ruled out as an underlying cause for the imitation deficit of facial movements, as it was not controlled for in the study. This might be particularly true for the upper face imitation tasks, as they contained five items regarding gaze movements. In one study in adults with HFA, a reduced eye region fixation time was found during viewing social scenes [29]. Therefore, reduced eye region fixation could have resulted in reduced upper facial movement imitation abilities in our study. 
Regarding differences in the imitation of lower facial movements, however, these are likely to be caused by a similar impairment as the reduced handand-finger gesture imitation abilities, as an association of lower facial movement imitation abilities with hand, finger and hand-and-finger gesture imitation abilities was found in the full sample, which was not present for upper facial imitation abilities. In one magnetoencephalography study on imitation of lip forms in AS, delayed activation of the inferior frontal lobule was found, whereas for the early activation in the STS and inferior parietal lobule, no differences were found [40]. Imitation difficulties in ASD might therefore be related to problems with top-down attentional control of spatial information [23]. However, this neuropsychological model has to be assessed in a more specific study designed to differentially elicit top-down (attentional, pre-frontal) and bottom-up (perceptual, temporal and parietal) processes and brain structures in face, hand and finger imitation. Also, a reduced integration of information between cortical regions involved in a common network for imitation of hand and finger gestures and facial movements [15, 35] might be a reason for the observed imitation deficits in ASD.

Differences in language abilities between ASD subjects and male and female controls were mainly found with regard to pragmatic aspects of language and the spontaneous speech measures of the AAT (communicative behaviour, articulation and prosody, semantic and syntactic structure). Pragmatic language impairments are a well-known feature of ASD [7]. Despite the strong differences in pragmatic abilities between groups, these were neither related to verbal or performance IQ measures nor to any of the imitation measures. Therefore, imitation abilities as assessed in our study might not be important for the development or maintenance of pragmatic language abilities. Pragmatic as well as semantic and syntactic tasks activated inferior and superior temporal gyri bilaterally in an fMRI study on healthy adults [30]. The temporal lobe has been involved in functional MRI or PET studies in ASD (e.g. $[14,16,56])$, therefore, the semantic, syntactic and pragmatic language problems might be primarily related to temporal lobe dysfunction in ASD. ASD subjects did not show signs of classical aphasia as would be indicated by differences in the token test. As in previous studies, subjects with ASD did not show any difficulties with comprehension or written language [42].

Contradictory to our hypotheses, correlations between imitation and language measures were not as strong as expected. This might partly be due to the low sample size and the differential correlation in the three comparison groups. Upper facial movements were correlated with the AAT spontaneous speech measure articulation and prosody, mainly due to a correlation in male controls. This correlation, however, was expected for lower facial movements, which did not show many correlations with expressive language abilities in our study. Of the hypothesized expressive language measures, only naming of articles, situations and actions was correlated with imitation abilities of lower facial movements, mainly due to a correlation in ASD subjects. This correlation might be explained by reduced language abilities mediated by the left inferior frontal gyrus (Broca's area) in ASD subjects. Broca's area has been found to be involved in syntactic [11] and semantic processing [49]. Reduced activation in this area compared to increased activation of Wernicke's areas was found in an fMRI study of sentence comprehension in HFA [28].

Female and male controls differed with regard to the association of non-meaningful hand and finger gestures with repetition of syllables, words and sentences. Imitation of hand gestures seems to be affected particularly in apractic patients with left temporo-parietal lesions [19]. Auditory word processing is mainly mediated by temporo-parietal areas as well, however, great individual variability exists regarding to additional activation of frontal areas [10]. This might be mediated by sex differences as well. Male ASD subjects did not show a similar pattern as male controls with regard to language and imitation abilities, arguing against the hypothesis of the extreme male brain theory [5].

In conclusion, ASD subjects showed problems with imitation of upper and lower face movements, of nonmeaningful hand-and-finger gestures and of AAT spontaneous speech measure as well as of pragmatic language abilities. The differential correlation of imitation and language measures in the three comparison groups might imply a differential organisation of language and imitation networks in the three groups. Pragmatic abilities, which are a central feature in ASD, were not related to imitation abilities. Therefore, imitation and language abilities in ASD might not be as closely correlated as previously expected.

\footnotetext{
Acknowledgements We thank the adolescents and their parents for participating in the study. Georg Goldenberg provided valuable advice for the design of the hand- and finger-imitation tasks. Rebecca Landa kindly made the Pragmatic rating scale - working draft available. The study was financially supported by the Saarland University, Saarbrücken, Germany.
} 


\section{References}

1. American Psychiatric Association (1994) Diagnostic and Statistical Manual of Mental Disorders, (4th edition) (DSM-IV). American Psychiatric Association, Washington DC

2. Achenbach TM (1991) Manual for the Youth Self-Report and 1991 Profile. Department of Psychiatry, University of Vermont, Burlington, VT

3. Achenbach TM (1997) Manual for the Young Adult Self-Report and Young Adult Behaviour Checklist. Department of Psychiatry, University of Vermont, Burlington, VT

4. Arbeitsgruppe Deutsche Child Behavior Checklist (1999) Deutsche Bearbeitung der Child Behavior Checklist (CBCL/4-18) - Einführung und Anleitung zur Handauswertung, 2. Auflage mit deutschen Normen. Koeln, Arbeitsgruppe Kinder-, Jugend- und Familiendiagnostik

5. Baron-Cohen S (2002) The extreme male brain theory of autism. Trends Cogn Neurosci 6:248-254

6. Bertone A, Mottron L, Jelenic P, Faubert J (2005) Enhanced and diminished visuo-spatial information processing in autism depends on stimulus complexity. Brain 128:2430-2441

7. Bishop DV, Baird G (2001) Parent and teacher report of pragmatic aspects of communication: use of the children's communication checklist in a clinical setting. Develop Med Child Neurol 43:809-818

8. Bizzozero I, Costato D, Della Sala S, Papagno C, Spinnler H, Venneri A (2000) Upper and lower face apraxia: role of the right hemisphere. Brain 123:2213-2230

9. Blake R, Turner LM, Smoski MJ, Pozdol SL, Stone WL (2003) Visual recognition of biological motion is impaired in children with autism. Psychol Sci 14:151-157

10. Burton MW, Noll DC, Small SL (2001) The anatomy of auditory word processing: individual variability. Brain Lang 77:119-131

11. Caplan D (2001) Functional neuroimaging studies of syntactic processing. J Psychol Res 30:297-320

12. Dawson G, Webb SJ, McPartland J (2005) Understanding the nature of face processing impairment in autism: insights from behavioural and electrophysiological studies. Develop Neuropsychol 27:403-424

13. Dakin S, Frith U (2005) Vagaries of visual perception in autism. Neuron 48:497-507
14. Freitag $C M$, Häberlen $M$, von Gontard A, Reith W, Krick C (2006) Imitation and recognition of biological motion in Autism Spectrum Disorders - a functional MRI study. (submitted)

15. Frith C (2003) What do imaging studies tell us about the neural basis of autism? Novartis Found Symp 251:149-166

16. Gervais H, Belin P, Boddaert N, Leboyer M, Coez A, Sfaello I, et al. (2004) Abnormal cortical voice processing in autism. Nat Neurosci 7:801-802

17. Ghiaziuddin M, Butler E (1998) Clumsiness in autism and Asperger syndrome: a further report. J Intel Disabilities Res 42:43-48

18. Gilchrist A, Green J, Cox A, Burton D, Rutter M, Le Couteur A (2001) Development and current functioning in adolescents with Asperger Syndrome: A comparative study. J Child Psychol Psych 42:227-240

19. Goldenberg G (1996) Defective imitation of gestures in patients with damage in the left or right hemispheres. J Neurol Neurosurg Psych 61:176-180

20. Goldenberg G, Hartmann K, Schlott I (2003) Defective pantomime of object use in left brain damage: apraxia or asymbolia? Neuropsychologia 41:15651573

21. Green D, Baird G, Barnett AL, Henderson $\mathrm{K}$, Huber J, Henderson SE (2002) The severity and nature of motor impairment in Asperger's syndrome: a comparison with Specific Developmental Disorder of Motor Function. J Child Psychol Psych 43:655-668

22. Hill EL, Frith U (2003) Understanding autism: insights from mind and brain. Phil Trans Royal Soc Lon B 358:281289

23. Hopfinger JB, Woldorff MG, Fletcher EM, Mangun GR (2001) Dissociating top-down attentional control from selective perception and action. Neuropsychologia 39:1277-1291

24. Howlin P (2003) Outcome in highfunctioning adults with autism with and without early language delays: implications for the differentiation between autism and Asperger Syndrome. J Autism Develop Disord 33:3-13

25. Huber W, Poeck K, Weniger D, Wilmes K (1983) Aachener Aphasie Test (AAT) - Handanweisung. Verlag für Psychologie Hogrefe, Göttingen, Toronto, Zürich

26. Iacoboni M, Woods RP, Brass M, Bekkering H, Mazziotta JC, Rizzolatti G (1999) Cortical mechanisms of human imitation. Science 286:2526-2528
27. Iacoboni M, Koski LM, Brass M, Bekkering $\mathrm{H}$, Woods $\mathrm{RP}$, Dubeau MC, Mazziotta JC, Rizzolatti G (2001) Reafferent copies of imitated actions in the right superior temporal cortex. Proc Natl Acad Sci USA 98:1399513999

28. Just MA, Cherkassky VL, Keller TA, Minshew NJ (2004) Cortical activation and synchronization during sentence comprehension in high-functioning autism: evidence of underconnectivity. Brain 127:1811-1821

29. Klin A, Jones W, Schultz R, Volkmar F, Cohen D (2002) Visual fixation patterns during viewing of naturalistic social situations as predictors of social competence in individuals with autism. Arch Gen Psych 59:809-816

30. Kuperberg GR, McGuire PK, Bullmore ET, Brammer MJ, Rabe-Hesketh S, Wright IC, Lythgoe DJ, Williams SC, David AS (2000) Common and distinct neural substrates for pragmatic, semantic, and syntactic processing of spoken sentences: an fMRI study. J Cog Neurosci 12:312-341

31. Koski L, Iacoboni M, Mazziotta JC (2002) Deconstructing apraxia: understanding disorders of intentional movement after stroke. Curr Opin Neurol 15:71-77

32. Landa R (2003) The Pragmatic Rating Scale - working draft, personal communication

33. Largo RH, Fischer J, Caflish J (2002) Zürcher Neuromotorik. AWE-Verlag, Zürich

34. Leiguarda RC, Marsden CD (2000) Limb apraxias. Higher-order disorders of sensorimotor integration. Brain 123:860-879

35. Leslie KR, Johnson-Frey SH, Grafton ST (2004) Functional imaging of face and hand imitation: towards a motor theory of empathy. NeuroImage 21:601-607

36. Lord C, Risi S, Lambrecht L, Cook EH, Leventhal BL, DiLavore PC, Pickles A, Rutter M (2000) The Autism Diagnostic Observation Schedule - Generic: a standard measure of social and communication deficits associated with the spectrum of autism. J Autism Develop Disord 30:205-223

37. Lord C, Rutter M, LeCouteur A (1994) Autism Diagnostic Interview - Revised: a revised version of a diagnostic interview for caregivers of individuals with possible pervasive developmental disorders. J Autism Develop Disord 24:659-685 
38. Meltzoff AN (1999) Origins of theory of mind, cognition and communication. J Commun Disord 32:251-269

39. Minshew NJ, Goldstein G, Siegel DJ (1997) Neuropsychologic functioning in autism: profile of a complex information processing disorder. J Intl Neuropsychol Soc 3:303-316

40. Nishitani N, Avikainen S, Hari R (2004) Abnormal imitation-related cortical activation sequences in Asperger's Syndrome. Annl Neurol 55:558-562

41. Oldfield RC (1971) The assessment and analysis of handedness: the Edinburgh inventory. Neuropsychologia 9:97-113

42. Ozonoff S, South M, Miller JN (2000) DSM-IV-defined Asperger Syndrome: cognitive, behavioural and early history differentiation from high-functioning autism. Autism 4:29-46

43. Papagno C, Della Sala S, Basso A (1993) Ideomotor apraxia without aphasia and aphasia without apraxia: the anatomical support for a double dissociation. J Neurol Neurosurg Psych 56:286-289

44. Pennington BF, Ozonoff S (1996) Executive functions and developmental psychopathology. J Child Psychol Psych 37:51-87
45. Plaisted K, Saksida L, Alcántara J, Weisblatt E (2003) Towards an understanding of the mechanisms of weak central coherence effects: experiments in visual configural learning and auditory perception. Philos Trans Roy Soc Lon B 358:375-386

46. Rogers SJ, Bennetto L, McEvoy R, Pennington BF (1996) Imitation and pantomime in high-functioning adolescents with autism spectrum disorder. Child Develop 67:2060-2073

47. Rogers SJ, Hepburn SL, Stackhouse T, Wehner E (2003) Imitation performance in toddlers with autism and those with other developmental disorders. J Child Psychol Psych 44:763-781

48. Rogers SJ, Pennington BF (1991) A theoretical approach to the deficits in infantile autism. Develop Psychopathol 3:137-163

49. Roskies AL, Fiez JA, Balota DA, Raichle ME, Peterson SE (2001) Task-dependent modulation of regions in the left inferior frontal cortex during semantic processing. J Cogn Neurosci 13:829-43

50. Smith IM, Bryson SE (1994) Imitation and action in autism: a critical review. Psychol Bull 116:259-273
51. Smith IM, Bryson SE (1998) Gesture imitation in autism I: Nonsymbolic postures and sequences. Cogn Neuropsychol 15:747-770

52. Stone WL, Ousley OY, Littleford CD (1997) Motor imitation in young children with autism: what's the object? J Abnorm Child Psychol 25:475-85

53. Stone WL, Yoder PJ (2001) Predicting spoken language level in children with autism spectrum disorders. Autism 5:341-361

54. World Health Organization (1992). The ICD-10 Classification of Mental and Behavioural Disorders. Clinical Description and Diagnostic Guidelines. World Health Organisation, Geneva

55. Williams JHG, Whiten A, Singh T (2004) A systematic review of action imitation in autistic spectrum disorder. J Autism Develop Disord 34:285-299

56. Zilbovicius $M$, Boddaert N, Belin P, Poline JB, Remy P, Mangin JF, Thivard K, Barthelemy C, Samson Y (2000) Temporal lobe dysfunction in childhood autism: a PET study. Amer J Psych 157:1988-1993

In the html abstract, html-text and pdf of the article

"Freitag CM et al. (2006) Imitation and language abilities in adolescents with Autism Spectrum Disorder without language delay. Eur Child Adolesc Psychiatry (published online March 22nd 2006)" The online version of the original article an be found at http://dx.doi.org/10.1007/s00787-006-0533-8

the name of the third author has been misspelled. Instead of

"Alexander von Gontardf"

it should have been

"Alexander von Gontard"

The publisher apologises for any inconvenience caused by this mistake. 